\title{
An iterative method for the dynamic modeling of ultra-short pulse generation in nonlinear optical ring resonator
}

\author{
Napoléon Gutierrez ${ }^{1}$, Arnaud Fernandez ${ }^{1}$, Olivier Llopis ${ }^{1}$, Sai Tak Chu ${ }^{2}$, Stéphane Balac ${ }^{3}$ \\ 1. LAAS-CNRS, Université de Toulouse, CNRS, UPS, Toulouse, France \\ 2. City University of Hong Kong, Tat Chee Avenue, Hong Kong SAR, China \\ 3. IRMAR, Université de Rennes 1, CNRS, Campus de Baulieu, 35042 Rennes, France
}

Numerical methods can be useful for the understanding of high-Q resonators, the interpretation of frequency comb formation and the tailoring of nonlinear phenomena. For specific applications like telecommunications or low phase noise microwave generation, a phase locked frequency comb or temporal pulse train is critical. Single intra-cavity dissipative temporal solitons have demonstrated to be attractive due to their stability and robustness. Femtosecond soliton generation has been obtained by red detuning an initially blue shifted CW pump laser through the cold cavity resonance of a non-linear resonator without requiring any feedback control [1]. Based on this outstanding result we have developed a numerical model that simulates this experimental setup for the prediction of the frequency comb build up at a resonator's output for a CW pump laser's set of parameters.

Our numerical approach takes benefit of RK4IP algorithm calculation efficiency for modeling the slowly varying complex envelope propagation inside the cavity through the generalized nonlinear Schrödinger equation [2]. We then couple, by the means of the optical coupler's transfer function, this cavity round trip result with the pump laser made up of a CW signal superimposed to white Gaussian noise. By numerically controlling the laser detuning and performing a frequency scan from the blue to red detuned regime over the cavity resonance we are able to visualize pattern formation and subsequently pulse formation (fig. 1 (a) and (b)). As opposed to the more conventional approach of using the Lugiato-Lefever model, our approach allows us to model the behaviour of any $\mathrm{CW}$ driven optical cavities without the need to resort to any averaging like said model [3]. This in turn allows for the accurate modeling of optical cavities of any given parameters, independently of their finesse.
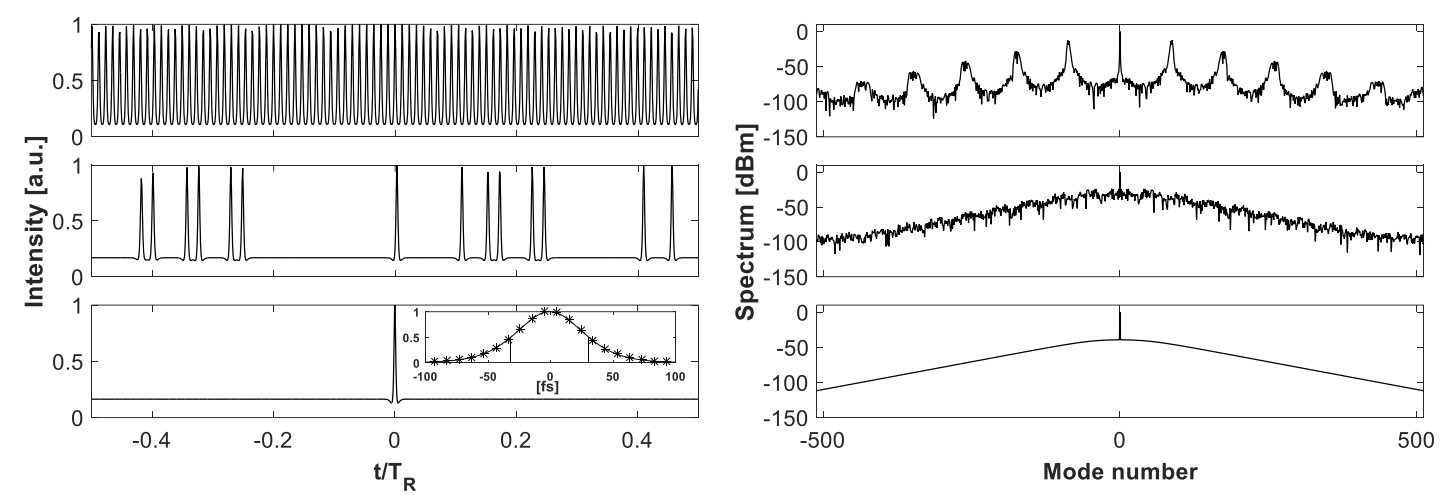

Fig. 1 Simulation results for a $50 \mathrm{GHz}$ FSR resonator with $\mathrm{Q}=10^{6}$ and a $100 \mathrm{~mW} \mathrm{CW}$ pump depicting different regimes. (a) Formation of temporal patterns inside the resonator. (b) Multiple soliton regime. (c) Single soliton mode-locked regime induced by pulse-like initial conditions inside the resonator. Inset shows the single soliton after filtering of the $\mathrm{CW}$ background. Approximation with an hyperbolic secant (markers) with a fitting error lower than $0.1 \%$.

Our numerical simulations were run with parameters corresponding to a $\mathrm{Q}=10^{6}$ high-index doped silica glass (Hydex) resonator with $50 \mathrm{GHz}$ free spectral range [4]. The random nature of the seeding of optical modes in a CW driven nonlinear optical cavity makes it so that each numerical scan simulation yields a different steady-state solution, all supported by our system [1]. By applying appropriate pulse-like initial conditions inside the resonator at a detuning that allows for the formation of solitons, we can guide the iterative method towards the steady-state solution we're interested in which is the single soliton regime. This spares us the need to run multiple scan simulations looking for this particular solution and lets us predict the behavior of our device.

\section{References}

[1] T. Herr et al., « Temporal solitons in optical microresonators », Nat. Photonics, vol. 8, n 2, p. 145-152, déc. 2013.

[2] S. Balac, A. Fernandez, F. Mahé, F. Méhats, et R. Texier-Picard, « The Interaction Picture method for solving the generalized nonlinear Schrödinger equation in optics », ESAIM Math. Model. Numer. Anal., vol. 50, nº 4, p. 945-964, juill. 2016.

[3] M. Haelterman, S. Trillo, et S. Wabnitz, « Dissipative modulation instability in a nonlinear dispersive ring cavity », Opt. Commun., vol. 91, nº 5-6, p. 401-407, août 1992.

[4] D. J. Moss, R. Morandotti, A. L. Gaeta, et M. Lipson, « New CMOS-compatible platforms based on silicon nitride and Hydex for nonlinear optics », Nat. Photonics, vol. 7, nº 8, p. 597-607, juill. 2013. 